\title{
Type Ia Supernova Models and Progenitor Scenarios
}

\author{
Ken'ichi Nomoto ${ }^{1,2, \dagger}$, Yasuomi Kamiya ${ }^{2,1}$, and Naohito Nakasato ${ }^{3}$ \\ ${ }^{1}$ Institute for the Physics and Mathematics of the Universe, University of Tokyo, \\ 5-1-5 Kashiwanoha, Kashiwa, Chiba 277-8583, Japan \\ ${ }^{2}$ Department of Astronomy, Graduate School of Science, University of Tokyo, \\ 7-3-1 Hongo, Bunkyo-ku, Tokyo 113-0033, Japan \\ ${ }^{3}$ Department of Computer Science and Engineering, University of Aizu, \\ Aizu-Wakamatsu, Fukushima 965-8580, Japan \\ ${ }^{\dagger}$ email: nomoto@astron.s.u-tokyo.ac.jp
}

\begin{abstract}
We review some recent developments in theoretical studies on the connection between the progenitor systems of Type Ia supernovae (SNe Ia) and the explosion mechanisms. (1) DDsubCh: In the merging of double $\mathrm{C}+\mathrm{O}$ white dwarfs (DD scenario), if the carbon detonation is induced near the white dwarf (WD) surface in the early dynamical phase, it could result in the (effectively) sub-Chandrasekhar mass explosion. (2) DD-Ch: If no surface C-detonation is ignited, the WD could grow until the Chandrasekhar mass is reached, but the outcome depends on whether the quiescent carbon shell burning is ignited and burns $\mathrm{C}+\mathrm{O}$ into $\mathrm{O}+\mathrm{Ne}+\mathrm{Mg}$. (3) $\mathrm{SD}$ subCh: In the single degenerate (SD) scenario, if the He shell-flashes grow strong to induce a He detonation, it leads to the sub-Chandra explosion. (4) $S D$-Ch: If the He-shell flashes are not strong enough, they still produce interesting amounts of $\mathrm{Si}$ and $\mathrm{S}$ near the surface of the $\mathrm{C}+\mathrm{O}$ WD before the explosion. In the Chandra mass explosion, the central density is high enough to produce electron capture elements, e.g., stable ${ }^{58} \mathrm{Ni}$. Observations of the emission lines of $\mathrm{Ni}$ in the nebular spectra provides useful diagnostics of the sub-Chandra vs. Chandra issue. The recent observations of relatively low velocity carbon near the surface of SNe Ia provide also an interesting constraint on the explosion models.
\end{abstract}

Keywords. nuclear reactions, nucleosynthesis, abundances — stars: supernovae, white dwarfs

\section{Introduction}

The observed features of Type Ia supernovae (SNe Ia) have been well-understood by a thermonuclear explosion of a carbon-oxygen $(\mathrm{C}+\mathrm{O})$ white dwarf (WD). Both the Chandrasekhar mass [Chandra $(\mathrm{Ch})$ model] and the sub-Chandrasekhar mass [sub-Chandra (subCh) model] have been presented (e.g., Livio 2000). However, there has been no clear observational indication as to how the WD mass grows until carbon ignition; i.e., whether the WD accretes $\mathrm{H} / \mathrm{He}$-rich matter from its binary companion [single degenerate $(\mathrm{SD})$ scenario], or two $\mathrm{C}+\mathrm{O}$ WDs merge [double degenerate (DD) scenario] (e.g., Hillebrandt \& Niemeyer 2000, Nomoto et al. 1997, 2000, 2009, Arnett 1996). Even before these issues are resolved, several candidates of super-Chandrasekhar mass explosions have been observed (e.g., Hachisu et al. 2011 and references therein).

Recent modeling shows that DD merging could result in both the Chandra and the (effectively) sub-Chandra explosions. The SD scenario could also result in both the Chandra and sub-Chandra explosions. Here we review such cross connections between (DD, SD) scenarios and (Chandra, sub-Chandra) models, and some observational constraints.

(1) DD-subCh: In the DD scenario, if the carbon detonation is induced near the WD surface in the early dynamical phase, it could result in the (effectively) sub-Chandra 
explosion. (2) DD-Ch: If no detonation is induced, the WD could grow until the Chandrasekhar mass is reached. The outcome depends on whether the quiescent carbon shellburning is ignited and burns interior $\mathrm{C}+\mathrm{O}$ into $\mathrm{O}+\mathrm{Ne}+\mathrm{Mg}$. (3) SD-subCh: In the SD scenario, if the He shell-flashes grow strong enough to induce a He detonation, it leads to a sub-Chandra explosion. (4) SD-Ch: If the He-shell flashes are not strong enough to induce a He detonation, such flashes produce interesting amount of intermediate mass elements, including Si and S, as unburned material near the surface of C+O WD.

\section{2. $\mathrm{C}+\mathrm{O}$ Double Degenerates to Sub-Chandra Mass Explosion}

In the DD scenario (Iben \& Tutukov 1984, Webbink 1984), two C+O WDs form a close binary system after the common envelope phase and get closer by losing orbital angular momentum due to gravitational radiation. Eventually, the less massive WD with a mass $M_{2}$ fills its Roche lobe and dynamically evolves to form a massive disk/envelope around the more massive WD with a mass $M_{1}$.

Such dynamical evolution of a WD binary has been studied by a number of authors (Benz et al. 1990, Segretain et al. 1997, Guerrero et al. 2004, Shioya et al. 2007, Pakmor et al. 2010) using the smoothed particle hydrodynamics (SPH) method.

Surface Carbon Detonation: The important question is whether the merging process ignites a surface carbon detonation in the WD. During the early merging process, the shock heating increases the temperature in the surface $\mathrm{C}+\mathrm{O}$ layer of the primary (i.e., more massive) WD. If the temperature becomes high enough to induce C-detonation (Pakmor et al. 2010 adopted the critical temperature of $2.5 \times 10^{9} \mathrm{~K}$ ), the detonation wave propagates through the central region of the primary WD. Eventually, the whole primary WD is detonated and disrupted.

This model is essentially the sub-Chandra mass explosion, because the detonated WD has a sub-Chandra mass of $M_{1}=0.9-1.1 M_{\odot}$ and the central density is as low as $\sim 10^{7} \mathrm{~g} \mathrm{~cm}^{-3}$ (Pakmor et al. 2010, 2011). The explosion produce a larger amount of ${ }^{56} \mathrm{Ni}$ for larger $M_{1}$.

\section{Double Degenerates to Chandra Mass Explosion or Collapse}

Suppose that surface C-detonation is not triggered in the early dynamical phase of merging. Then the next important question is whether the merging process ignites not the C-detonation but "quiescent" off-center carbon burning in the WD. Once carbon is ignited, carbon flame moves inward by heat conduction to reach the center (Saio \& Nomoto 1985, 1998). The released nuclear energy is lost in neutrinos, and the $\mathrm{C}+\mathrm{O}$ $\mathrm{WD}$ is converted into the $\mathrm{O}+\mathrm{Ne}+\mathrm{Mg} \mathrm{WD}$ non-explosively. The ONeMg WDs eventually collapse due to electron capture rather than exploding as SNe Ia (Nomoto 1984, 1987).

\subsection{Carbon Shell Burning and Chandra Explosion or Collapse}

Here the carbon ignition temperature $T_{\text {ign }}$ is defined by $\epsilon_{\mathrm{C}+\mathrm{C}}=\epsilon_{\nu}$, where $\epsilon_{\mathrm{C}+\mathrm{C}}$ and $\epsilon_{\nu}$ denote the nuclear energy generation rate and the neutrino energy loss rate, respectively. At $\rho \sim 1-3 \times 10^{6} \mathrm{~g} \mathrm{~cm}^{-3}, T_{\text {ign }} \sim 6 \times 10^{8} \mathrm{~K}$ (Nomoto \& Iben 1985, Kawai et al. 1987, Yoon et al. 2007).

For $T>T_{\mathrm{ign}}, \epsilon_{\mathrm{C}+\mathrm{C}}>\epsilon_{\nu}$ and the carbon flash is ignited, and the conductive carbon flame propagates inward. If $T<T_{\mathrm{ign}}$, on the contrary, $\epsilon_{\mathrm{C}+\mathrm{C}}<\epsilon_{\nu}$ and the neutrino cooling dominates to induce the gradual contraction of the C-rich envelope. Then a carbon flame is not formed. 


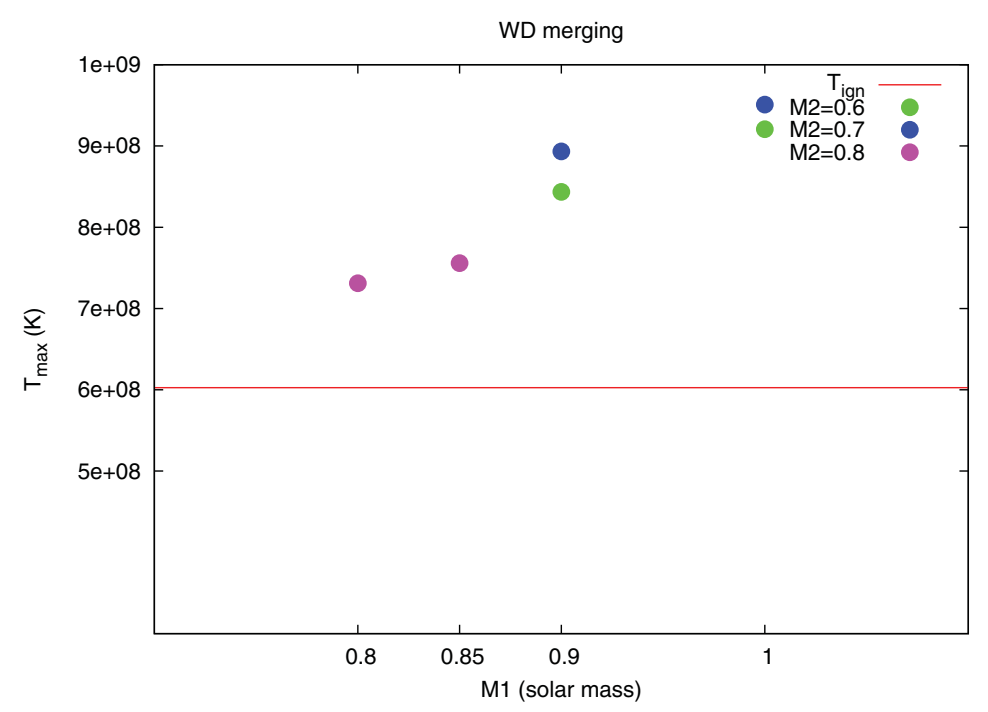

Figure 1. The local peak temperature $T_{\text {peak }}$ attained 2.5 min after the start of merging of double $\mathrm{C}+\mathrm{O}$ white dwarfs with masses $\left(M_{1}, M_{2}\right)$ (Nakasato \& Nomoto 2011). For $T_{\text {peak }}>T_{\mathrm{ign}}$, off-center carbon burning is ignited.

There are two possible cases for off-center carbon ignition to occur.

Case 1: When the falling materials from the less massive star compress the outer layer of the more massive star, the material could be heated up to high enough temperature to ignite carbon burning.

Case 2: Later, if the accretion rate exceeds a critical rate of $2.7 \times 10^{-6} M_{\odot} \mathrm{yr}^{-1}$, compressional heating exceeds radiative cooling and leads to carbon ignition (Nomoto \& Iben 1985, Kawai et al. 1987).

For Case 1, Yoon et al. (2007) calculated the merging process until 5 min after its start and showed that a quasi-static equilibrium configuration is reached consisting of a cold core, hot envelope, and a disk. The peak temperature $T_{\text {peak }}$ reaches a stationary value. For $M_{1}=0.9 M_{\odot}$ and $M_{2}=0.6 M_{\odot}, T_{\text {peak }}$ is marginally lower than $T_{\text {ign }}$. Whether the later off-center carbon ignition of Case 2 takes place depends on the effective accretion rate, which needs further study (e.g., Shioya et al. 2007, Shen et al. 2011).

For Case 1, Nakasato \& Nomoto (2011) have recently conducted SPH simulations (Nakasato \& Nomoto 2003) with the number of particles $N=300,000$ and $N=$ $1,000,000$ for various combinations of $\left(M_{1}, M_{2}\right)$. The artificial viscosity is treated to minimize numerical effects according to the hybrid scheme proposed by Rosswog et al. (2010). The Helmholtz equation of state is used (Timmes \& Swesty 2000), and the WD is assumed to be composed of $50 \%$ carbon and $50 \%$ oxygen.

The local peak temperatures shown in Figure 1 are obtained from particles at $\rho \sim 1-$ $3 \times 10^{6} \mathrm{~g} \mathrm{~cm}^{-3}$ at $2.5 \mathrm{~min}$ after the start of merging. $T_{\text {peak }}$ has already reached its stationary value, which is confirmed with some test runs calculated until 10 min after the merging. It is seen that $T_{\text {peak }}$ is determined mainly by $M_{1}$ with small dependence on $M_{2}$. This implies that the gravitational potential of the more massive WD is the main factor to determine $T_{\text {peak }}$.

Figure 1 shows that $T_{\text {peak }}>T_{\text {ign }}$ for most sets of values of $\left(M_{1}, M_{2}\right)$. Thus, a carbon flash will take place to form a carbon flame that propagates inward to convert $\mathrm{C}+\mathrm{O}$ into $\mathrm{O}+\mathrm{Ne}+\mathrm{Mg}$. 


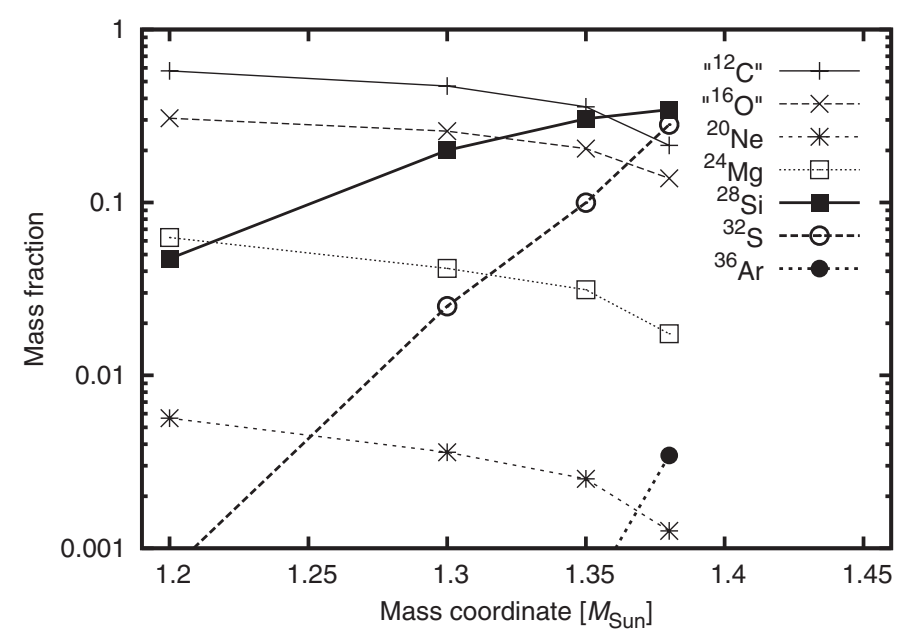

Figure 2. Abundance distribution of the products of He shell flashes in an accreting WD at the explosion (Kamiya \& Nomoto 2011).

How do the above results depend on the set-up of the merging calculations (Dan et al. 2011)? The initial separation is set to be $a=\left(0.9 R_{2}\right) / r_{\mathrm{L}}$ where $R_{2}$ is the radius of the less massive WD and $r_{\mathrm{L}}$ is the effective Roche lobe radius. Some test runs adopting a larger separation confirm that $T_{\text {peak }}$ is not sensitive to the initial separation.

Comparisons between the runs with $N=300,000$ and with $N=1,000,000$ show that $T_{\text {peak }}$ of the higher resolution run is always slightly higher than the low resolution run. Thus carbon ignition of Case 1 is quite likely for most cases of $\left(M_{1}, M_{2}\right)$.

\subsection{Further Evolution of Rotating White Dwarfs}

The WD formed from merging must be rapidly rotating. The "SN Ia mass" of the WD with which an SN Ia is triggered is $M_{\text {Ia }}=1.48 M_{\odot}$ for a uniformly rotating WD at the critical rotation (Uenishi et al. 2003). This is larger than $1.38 M_{\odot}$ for the non-rotating WD. For non-uniform, differentially rotating WDs, $M_{\text {Ia }}$ is as large as $\gtrsim 2 M_{\odot}$ (Yoon \& Langer 2004, Hachisu et al. 2011). Therefore, even if no carbon flame is formed, the WD may not reach $M_{\text {Ia }}$ because of some mass loss after merging.

\section{Single Degenerate to Sub-Chandra Explosion}

In the SD scenario, H-burning produces a thin He layer, and He-flashes are ignited when the He mass reaches a certain critical value. The strength depends on the He envelope mass $M_{\text {env }}$, thus depending on the accretion rate.

The He envelope mass $M_{\text {env }}$ is larger for the slower mass-accumulation rate of the $\mathrm{He}$ layer $\dot{M}_{\mathrm{He}}$. For $\dot{M}_{\mathrm{He}} \gtrsim 1 \times 10^{-8} M_{\odot} \mathrm{yr}^{-1}, M_{\text {env }}$ exceeds a critical value where the density at its bottom becomes high enough to induce a He detonation. This would result in the sub-Chandra explosion (e.g., S. Sim in this conference).

\section{Single Degenerate to Chandra Mass Explosion}

For higher $\dot{M}_{\mathrm{He}}$, the He-shell flashes are not strong enough to induce a He detonation. Then, such flashes repeat many times with the increasing WD mass $M_{\mathrm{WD}}$ toward the Chandrasekhar mass. Kamiya \& Nomoto (2011) calculated nucleosynthesis in such He shell flashes for various set of $\left(M_{\mathrm{WD}}, M_{\mathrm{env}}\right)$ (see also Shen \& Bildsten 2007). 

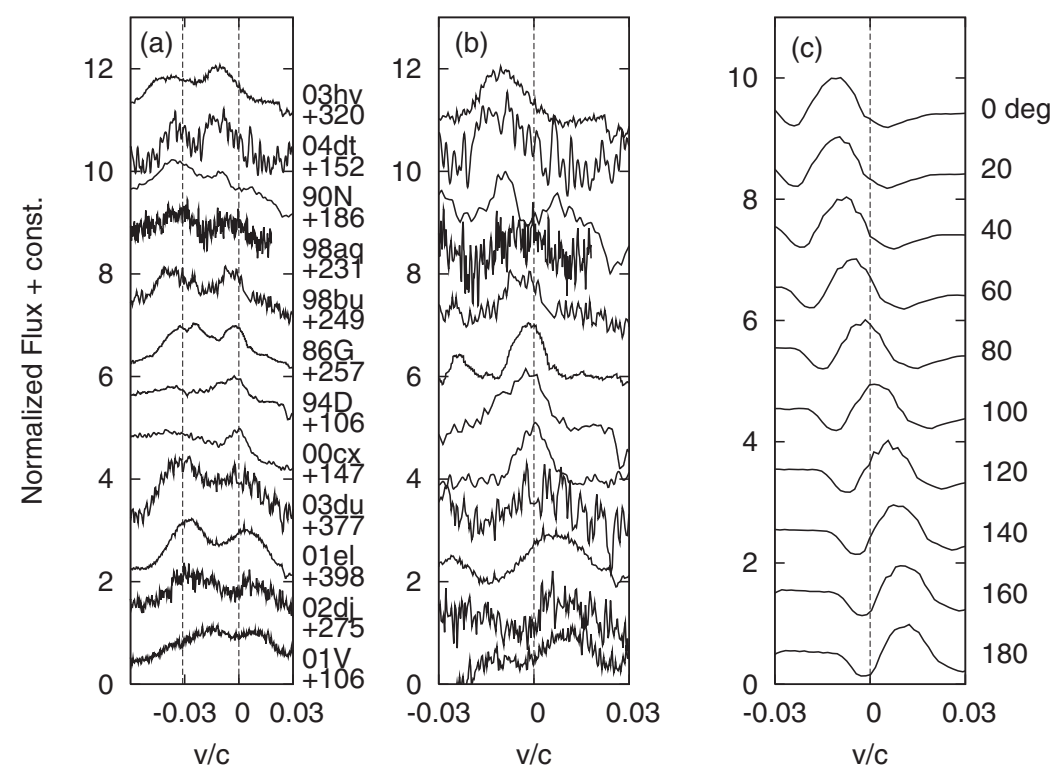

Figure 3. Analysis of the [Ni II] $\lambda 7378$ line profiles in 12 SNe Ia (Maeda et al. 2010a). The velocity is set assuming that the rest wavelength is at $7378 \AA$. (a) Observed line profiles. The rest wavelengths of [Fe II] $\lambda 7155$ and [Ni II] $\lambda 7378$ are shown by dotted lines. (b) [Ni II] $\lambda 7378$ in observations, after removing the underlying continuum (or possible other lines). (c) Synthetic line profiles of the [Ni II], depending on the viewing orientation.

For $\dot{M}_{\mathrm{He}} \sim(0.5-1) \times 10^{-7} M_{\odot} \mathrm{yr}^{-1}$, the WD is expected to undergo He shell-flashes at $\left(M_{\mathrm{WD}} / M_{\odot}, \log \left(M_{\mathrm{env}} / M_{\odot}\right)\right) \sim(1.2,-2.5),(1.3,-3),(1.35,-3.5),(1.38,-4)$ as $M_{\mathrm{WD}}$ grows (Kato et al. 2008).

In the early stages of the He shell-flash, the envelope is electron-degenerate and geometrically almost flat. Thus the temperature at the bottom of the He-burning shell increases because of the almost constant pressure there. Heated by nuclear burning, the helium envelope gradually expands, which decreases the pressure. Then, the temperature attains its maximum and starts decreasing. The maximum temperature is higher for more massive WDs and more massive envelopes because of the higher pressure.

For higher maximum temperatures, heavier elements such as ${ }^{28} \mathrm{Si}$ and ${ }^{32} \mathrm{~S}$ are synthesized. However, the maximum temperature is not high enough to produce ${ }^{40} \mathrm{Ca}$. After the peak, some amount of He remains unburned in the flash and burns into $\mathrm{C}+\mathrm{O}$ during the stable He shell burning.

In this way, it is possible that interesting amounts of intermediate mass elements, including $\mathrm{Si}$ and $\mathrm{S}$, already exist in the unburned $\mathrm{C}+\mathrm{O}$ layer at $M_{r} \geqslant 1.2 M_{\odot}$. Such a distribution is shown in Figure 2 for $\dot{M}_{\mathrm{He}} \sim 5 \times 10^{-8} M_{\odot} \mathrm{yr}^{-1}$. A part of them might be ejected out. Therefore the above quantity of the synthesized elements are overestimated.

\section{Electron Capture in Chandra Mass Models}

Both Chandra and sub-Chandra explosion models can synthesize relevant amounts of ${ }^{56} \mathrm{Ni}$ for SNe Ia. However, the amount of other Fe-peak elements differs, because the ignition density is different, being as high as $>10^{9} \mathrm{~g} \mathrm{~cm}^{-3}$ in the Chandra model, while as low as $\sim 10^{7} \mathrm{~g} \mathrm{~cm}^{-3}$ in the sub-Chandra model.

In the Chandra model, the thermonuclear runaway starts with the ignition of deflagration (e.g., Nomoto et al. 1976, 1984). In the high temperature and density bubble, 


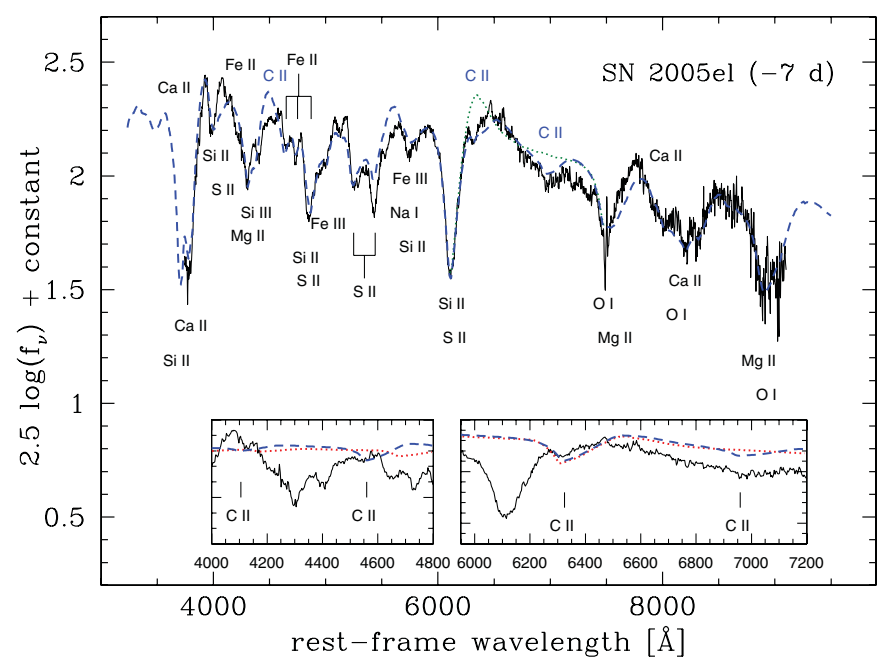

Figure 4. Spectrum of SN 2005el at -7 days (solid line), and a matching SYNOW calculation (dashed line). (Insets) The spectrum near the location of C II lines (solid line), the SYNOW spectra containing only C II (dashed line), and only H I (dotted line). The expected locations of C II lines are marked (Folatelli et al. 2011)

materials are incinerated into NSE (nuclear statistical equilibrium) and undergo electron capture. Electron capture on free protons and Fe-peak elements leads to the synthesis of ${ }^{58} \mathrm{Ni},{ }^{54} \mathrm{Fe}$, and ${ }^{56} \mathrm{Fe}$ (not via ${ }^{56} \mathrm{Ni}$ decay). These neutron-rich Fe-peak elements form an almost ${ }^{56} \mathrm{Ni}$-empty hole (e.g., Nomoto et al. 1984).

In the sub-Chandra model, the ignition density is too low for electron capture to take place. The neutron excess is produced only by the initial CNO elements which are converted to ${ }^{14} \mathrm{~N}$ and to ${ }^{22} \mathrm{Ne}$, thus depending on the initial metallicity. As a result, the mass fraction of ${ }^{58} \mathrm{Ni}$ is as small as $\sim 0.01$ (e.g., Shigeyama et al. 1992).

Such a difference in the amount of ${ }^{58} \mathrm{Ni}$ can be observationally investigated by latephase $(\sim 1$ yr since the explosion) spectroscopy at near-infrared (NIR) wavelengths. Because the ejecta become optically thin in late phases, spectroscopy provides an unbiased, direct view of the innermost regions.

Figure 3 shows the spectral feature around $\sim 7000-7500 \AA$, i.e., $[\mathrm{Fe}$ II] $\lambda 7155$ (with some contribution from [Fe II] $\lambda 7171$ ) and [Ni II] $\lambda 7378$, for 12 SNe Ia (Maeda et al. 2010a). The [Ni II] $\lambda 7378$ line is emitted from the electron capture region of the ejecta, which is supported by the relatively narrow width $\left(\lesssim 3,000 \mathrm{~km} \mathrm{~s}^{-1}\right)$ of the [Ni II] line. Thus the existence of [Ni II] line implies the ignition at high density, thus favoring the Chandra model.

It is also interesting to note that these emission lines show the velocity shift, which indicates the off-center ignition and aspherical nature of SNe Ia explosions (Maeda et al. 2010a, 2010b). The aspherical features could be seen in the light echo from Tycho's supernova remnant (Usuda et al. 2011).

\section{Carbon in SNe Ia}

Parrent et al. (2011) and Folatelli et al. (2011) investigated the presence of unburned material in early-time spectra of SNe Ia. They find that at least $30 \%$ of the objects in the sample show absorption at about $6300 \AA$ which can be associated with C II $\lambda 6580$ (Figure 4). This would imply a larger incidence of carbon in SNe Ia ejecta than 
previously noted. If confirmed as carbon, the material producing the observed features would be present at very low expansion velocities, merely $\sim 1,000 \mathrm{~km} \mathrm{~s}^{-1}$ above the Si II velocities.

Carbon must be present in very deep regions, corresponding to velocities as low as $v \approx$ $11,000 \mathrm{~km} \mathrm{~s}^{-1}$. This is well below the expected limit imposed by one-dimensional models, and points directly to large mixing effects and/or possible departures from spherical symmetry or clumpiness.

In spherically symmetric models, irrespective of the details of the flame propagation (deflagration or detonation), the production of a large amount of ${ }^{56} \mathrm{Ni}\left(\sim 0.6 M_{\odot}\right)$ requires that the strength of the flame must lead to the total consumption of carbon below 15,000 - 20,000 $\mathrm{km} \mathrm{s}^{-1}$ (Nomoto et al. 1984, Shigeyama et al. 1992, Iwamoto et al. 1999). The situation is different for non-spherical models. For example, the off-center ignition model (Maeda et al. 2010a; see also Kasen et al. 2009) synthesizes $0.54 M_{\odot}$ of ${ }^{56} \mathrm{Ni}$ and carbon with a mass fraction of $\sim 0.1$ at velocities as low as $\sim 13,000 \mathrm{~km} \mathrm{~s}^{-1}$. This is still larger than the observed velocity, but suggests that the non-spherical effects may be important to understand the detection of carbon deep in the ejecta.

The existence of carbon at relatively low velocity suggests the presence of fair amount of unburned $\mathrm{C}+\mathrm{O}$ materials. The amount of $\mathrm{Fe}$ peak elements in such an unburned layer depends on the metallicity. Further observations of early UV spectra could show significant metallicity effects.

\section{Concluding Remarks}

As summarized above, recent modeling shows that DD merging could result in both the Chandra and the sub-Chandra explosions depending on whether a carbon detonation is induced near the surface of more massive WDs. The SD scenario could also result in both Chandra and sub-Chandra explosions depending on whether the He shell-flashes near the surface of the WD induce a He detonation.

- For DD-subCh, it is critically important to confirm the formation of surface carbon detonation by means of 3D hydrodynamical simulations rather than SPH method.

- For DD-Ch, whether carbon ignition can occur for both Case 1 and Case 2 needs further study for various sets of $\left(M_{1}, M_{2}\right)$.

- For SD-subCh, a mechanism to avoid the production of too much ${ }^{56} \mathrm{Ni}$ in the surface should be studied. In case of the He-WD and C-WD merger, formation of an extended He envelope needs to be avoided.

- For SD-Ch, the outcome of quiescent He-shell flashes, e.g., nucleosynthesis, the rate of the He wind mass loss, needs to be studied.

- Finally, detailed hydrodynamical modeling of the WD spin-down is necessary. In this spin-down scenario (e.g., Justham 2011, Di Stefano et al. 2011, Ilkov \& Soker 2011, Hachisu et al. 2011), an almost uniformly rotating $\mathrm{C}+\mathrm{O}$ WD with a mass range of 1.38 $1.48 M_{\odot}$ forms and eventually contract to ignite carbon after a long spin-down time. The outcome depends on the ignition density. If it is as high as $\sim 10^{10} \mathrm{~g} \mathrm{~cm}^{-3}$, electron capture induces collapse rather than explosion (Nomoto \& Kondo 1991). If it is lower, an SN Ia explosion would result.

This research has been supported in part by World Premier International Research Center Initiative, MEXT, Japan, and by the Grant-in-Aid for Scientific Research of the JSPS (23540262) and MEXT (22012003, 23105705). 


\section{References}

Arnett, W. D. 1996, Nucleosynthesis and Supernovae (Princeton: Princeton Univ. Press)

Benz, A. O., Cameron, A. G. W., Press, W. H., \& Bowers, R. L. 1990, ApJ, 348, 647

Dan, M., Rosswog, S., Guillochon, J., \& Ramirez-Ruiz, E. 2011, ApJ, 737, 89

Di Stefano, Voss, R., \& Claeys, J. S. W. 2011, ApJ, 738, L1

Folatelli, G., Phillips, M. M., Morrell, N., Tanaka, M., Maeda, K., Nomoto, K., et al. 2011, ApJ, submitted

Guerrero, J., Garcis-Berro, E., \& Isern, J. 2004, A\&A, 413, 257

Hachisu, I., Kato, M., Saio, H., \& Nomoto, K. 2011, ApJ, in press (arXiv:1106.3510)

Hillebrandt, W. \& Niemeyer, J. 2000, ARAA, 38, 191

Ilkov, M. \& Soker, N. 2011, arXiv:1106.2027

Iwamoto, K., Nomoto, K., \& Thielemann, F.-K. 1999, ApJS, 54, 335

Iben, I., Jr. \& Tutukov, A. V. 1984, ApJS, 54, 335

Justham, S. 2011, ApJ, 730, L34

Kasen, D., Röpke, F., \& Woosley, S. 2009, Nature, 460, 869

Kamiya, Y. \& Nomoto, K. 2011, in preparation

Kato, M., Hachisu, I., Kiyota, S., \& Saio, H. 2008, ApJ, 684, 1366

Kawai, Y., Saio, H., \& Nomoto, K. 1987, ApJ, 315, 229

Livio, M. 2000, Type Ia Supernovae: Theory and Cosmology (Cambridge Univ. Press), 33

Maeda, K., et al. 2010a, ApJ, 394, 239

Maeda, K., et al. 2010b, Nature, 466, 82

Nakasato, N. \& Nomoto, K. 2003, ApJ, 588, 842

Nakasato, N. \& Nomoto, K. 2011, in preparation

Nomoto, K. 1984, ApJ, 277, 791

Nomoto, K. 1987, ApJ, 322, 206

Nomoto, K. \& Iben, I. Jr. 1985, ApJ, 297, 53

Nomoto, K., Iwamoto, K., \& Kishimoto, N. 1997, Science, 276, 1378

Nomoto, K., Kamiya, Y., Nakasato, N., Hachisu, I., \& Kato, M. 2009, in AIPC 1111: Probing Stellar Populations out to the Distant Universe: Cefalu 2008 (AIP), 267

Nomoto, K. \& Kondo, Y. 1991, ApJ, 367, L19

Nomoto, K., Sugimoto, D., \& Neo, 1976, Ap. Sp. Sci., 39, L37

Nomoto, K., Thielemann, F.-K., \& Yokoi, K. 1984, ApJ, 286, 644

Nomoto, K., Umeda, H., Kobayashi, C., Hachisu, I., Kato, M., \& Tsujimoto, T. 2000, in AIPC 522: Cosmic Explosions, ed. S. S. Holt \& W. W. Zhang (AIP), 35 (astro-ph/0003134)

Pakmor, R., et al. 2010, Nature, 463, 61

Pakmor, R., et al. 2011, Supernovae and their Host Galaxies, Sydney 2011

Parrent, J. T., et al. 2011, ApJ, 732, 30

Rosswog, S., Davis, M. B., Thielemann, F.-K., \& Piran, T. 2000, A\&A, 360, 171

Saio, H. \& Nomoto, K. 1985, A\&\&A, 150, L21

Saio, H. \& Nomoto, K. 1998, ApJ, 500, 388

Segretain, L., Chabrier, G., \& Mochkovitch, R. 1997, ApJ, 481, 355

Shen, K. \& Bildsten, L. 2007, ApJ, 660, 1444

Shen, K., Bildsten, L., Kasen, D., \& Quataert, E. 2011, ApJ, submitted (arXiv:1108.4036)

Shigeyama, T., Nomoto, K., Yamaoka, H. \& Thielemann, F.-K. 1992, ApJ, 386, L13

Shioya, T., Sano, T., \& Takabe, H. 2007, PASJ, 59, 753

Timmes, F. X. \& Swesty, F. D. 2000, ApJS, 126, 501

Uenishi, T., Nomoto, K., \& Hachisu, I. 2003, ApJ, 595, 1094

Usuda, T., Krause, O., Tanaka, M., Hattori, T., Goto, M., Birkmann, S. M., \& Nomoto, K. 2011, in these Proceedings

Webbink, R. F. 1984, ApJ, 277, 355

Yoon, S.-C. \& Langer, N. 2004, A\&A, 419, 623

Yoon, S.-C., Podsiadlowski, Ph., \& Rosswog, S. 2007, MNRAS, 380, 933 